\title{
Association between device measured and self- reported sitting in relation to depression: the 1970 British Cohort Study
}

\author{
Andrew Webster \\ UCL \\ G David Batty \\ University College London \\ Natalie Pearson \\ Loughborough University \\ Emmanuel Stamatakis \\ UNIVERSITY OF SYDNEY \\ Mark Hamer ( $\square$ m.hamer@ucl.ac.uk) \\ University College London
}

\section{Research}

Keywords: Depression, Sedentary Behaviour, Device-Measured, Objectively Measured, Sitting Time, BCS70

Posted Date: March 15th, 2020

DOl: https://doi.org/10.21203/rs.3.rs-17236/v1

License: (c) (i) This work is licensed under a Creative Commons Attribution 4.0 International License. Read Full License 


\section{Abstract}

Aims While physical activity appears to confer protection against depression, the relationship between sedentary behaviour and mental health is uncertain. Self-reported methods provide information about context although there may be error in the quantification of sedentary behaviour. Accordingly, we examined associations of both device-measured and self-reported sedentary behaviour with depression.

Method Participants ( $n=4,704 ; 52.4 \%$ Female; aged 46-48) were drawn from the 1970 British Cohort Study. Sitting time and moderate-vigorous physical activity was measured using a thigh-worn accelerometer device (ActivPAL) over a seven day period. A range of self-reported sedentary behaviours was measured to provide context. Depression diagnosis was captured using a combination of self-reported consultation with a physician and use of anti-depressant medication. Malaise inventory was used to assess depressive symptoms.

Results Relative to those who spent $<8 \mathrm{hr} / \mathrm{d}$ sitting, those in the highest tertile of device measured sitting $(>10 \mathrm{hr} / \mathrm{d}$ ) had increased odds of depression diagnosis (odds ratio= 1.48 [95\% confidence interval 1.052.08]). There was no association between self-reported TV viewing and depression diagnosis (1.07; 0.711.63). We observed protective associations between moderate-vigorous physical activity and depression diagnosis (highest tertile vs. the lowest tertile; 0.70;0.49-1.00). Associations of sitting time and physical activity with depression were mutually independent. Relative to $<1$ hours of internet usage, $2-3$ and $>3$ hours of internet weekday usage were associated with increased odds of depressive symptoms (1.60;1.30-1.97 and 1.63;1.32-2.03, respectively).

Conclusion Device-measured sitting is associated depression diagnosis, although less consistent associations are observed with self-reported sedentary behaviours. Regular physical activity and reducing sedentary time may be beneficial for prevention of depression.

\section{Introduction}

Common Mental Disorder (CMD) (depression and anxiety), are highly prevalent in the United Kingdom (UK) with one in six UK adults experiencing a mental health issue in any given week.[1] Furthermore it has been suggested that depression will be the world leading cause for disease burden and disability by 2030.[2] Whilst CMD has been highlighted as an important health outcome in it's own right, CMD has been linked to an increased risk of suicide, unintentional injury and chronic disease such as cardiovascular events and selected cancers. [3-6]

There is potential to prevent depression with regular physical activity (PA) and exercise. [7] Results from a recent meta-analysis of prospective cohort studies showed regular high levels of PA was associated with lowered odds of future depressive symptoms irrespective of age and sex. [8] However, evidence for an association between sedentary behaviour (SB) and depression is less well established. SB is defined as "Any waking behaviour characterized by an energy expenditure $\leq 1.5$ Metabolic Equivalents (METs), while in a sitting, reclining or lying posture."[9] The existing evidence is largely based on observational data,[10,11] 
although evidence from a recent randomised controlled trial observed a significant increase in depressive mood when young physically active adults were placed under experimental sedentary conditions.[12]

In most studies SB has been assessed using self-reported methods. Whilst the utilisation of self-reported SB is convenient for large scale data collection and does provide important information on context, the method suffers from reporting bias.[13] Indeed, reporting bias may be partly driven by depressive symptoms as people with low mood may respond differently to questions about their lifestyle.[14] This could explain why there are some inconsistent and contrasting findings on the links between SB and depression.[15-17] Wearable devices yield more valid and reliable measurement of SB when compared to a self-report questionnaire.[18] However, accelerometery does not capture SB context that may provide important information.

To the best of our knowledge, no large scale, population-based cohort study has utilised the gold standard thigh-mounted accelerometery approach to examine associations between sitting time and depression. The aims of the current study therefore were to investigate associations of device measured sitting and selfreported sedentary behaviours with depression in a population sample of British adults.

\section{Method}

\section{Sample and Study Design}

The 1970 British Cohort Study (BCS70)follows the lives of people born in 1970 in a single week in England, Scotland and Wales. [19] For the present cross-sectional study we utilised data from the age 46 sweep of BCS70. Data was collected during a home visit conducted in 2016-18, which compromised of 50 minutes interviews (face-to-face, computer-assisted-personal-interview and computer-assisted-self-completion interviews) with a further 50 minutes of biomedical assessments performed by trained nurses.[20] Participants provided informed consent and the study received full ethical approval from NRES Committee South East Coast - Brighton \& Sussex (Ref 15/LO/1446).

\section{Objectively measured Physical Activity and Sedentary Behaviour}

Cohort members were asked to wear the activPAL thigh mounted accelerometer (activPAL3 micro; PAL Technologies Ltd., Glasgow, UK), to measure both sitting and physical activity levels. The device uses derived information about thigh inclination and acceleration to estimate body posture (sitting/lying and upright) and transition between these postures, stepping, and stepping speed (cadence).[21-25] ActivPAL was validated for measuring free-living sedentary behaviour against direct observation using an automated camera (Youden's Index: 92.48 [87.26-97.70]). [26] The thigh mounted device helps overcome established limitations of a hip-worn accelerometer, which are known to misclassify sitting and standing. [18]

The activPAL was set to record at the sampling frequency of $20 \mathrm{~Hz}$ in accordance with other wear-time protocols.[27] The device was positioned on the midline anterior aspect of the upper thigh by the research nurses during the home visit, and was waterproofed, in line with the manufacturer's recommendations. 
During the seven-day consecutive wear protocol, the cohort members were instructed to wear the device when performing any normal activities of daily living including exercise, swimming and bathing, and instructed not to reattach the device if removed. Data were processed using freely available software that has been previously validated with an almost perfect agreement $(k>0.8)$ and a median Kappa of 0.94 (https://github.com/UOL-COLS/ProcessingPAL/releases/tag/V1.0).[28] The software uses an algorithm to isolate valid waking wear data from sleep or prolonged non-wear, summarized elsewhere. [28] MVPA was calculated using a step cadence $\geq 100$.[29] Valid data was classified as 10 hours of waking wear time for at least one day.

\section{Contextual Sedentary Behaviour}

Cohort members reported on a variety of different contexts of SB. This included on average how many hours were spent viewing TV and internet usage on a typical week day. Furthermore, participants were asked to rate their level of occupational physical activity using four response options (sitting; standing, physically active; heavy manual work occupations).

\section{Depression}

Cohort members were asked if they had seen a doctor regarding depression and if they had used antidepressant medication. Data on the usage of prescribed antidepressants was obtained from medication dispensers during the nurse's visit, using the British National Formulary edition 69 code. Depression diagnosis was indicated by a positive response to both enquiries given that anti-depressants may in some instances be prescribed for conditions other than depression. Study members were also asked about symptoms of depression using the Malaise Inventory.[30] This 9-item scale has acceptable psychometric properties when compared to clinical review of the interview ratings from an experienced psychiatrist, with a receiver operating characteristic score of 0.87 for both case level depression and any current affective disorder.[31] Overall, the Malaise Inventory has good validity within the adult general population with high levels of internal reliability over 10 years between ages 23 and 33 ( 0.77 and 0.80 respectively). It has also been shown to have a moderate stability score over a period of 10 years of $r=0.52$.[32] The Malaise Inventory was scored from $0-9$, with a greater score denoting a greater severity of depressive mood. A Malaise Inventory Score of $\geq 4$ was used to indicate the presence of elevated depressive symptoms. [33]

\section{Covariates}

Covariates were identified a priori from existing literature and included sex (male or female), device waking hours wear time, cigarette smoking (never smoked, ex-smoker, occasional and daily smoker), alcohol consumption (never, monthly, weekly), body mass index (underweight $\left[<18.5 \mathrm{~kg} / \mathrm{m}^{2}\right]$, normal weight [18.5$24.9 \mathrm{~kg} / \mathrm{m}^{2}$ ], overweight [25.0-29.9 kg/m²], obese [ $\left.\geq 30 \mathrm{~kg} / \mathrm{m}^{2}\right]$ ), disability using European Union-statistics on income and living condition [EU-SILC] classification (none; some extent, severely hampered), education (no qualifications, secondary school education [GCSE and A-Levels and Diplomas], graduate education [BSc, $\mathrm{MSc}, \mathrm{PhD}]$ ). For analyses using device measures of sitting we additionally adjusted for device measured MVPA (categorised into roughly equal tertiles: $\leq 0.6,0.6-0.9, \geq 0.9$ hours,), although in analyses using selfreported data we adjusted for self-reported frequency of weekly exercise bouts, consisting of 30 minutes or more $(0-1,2-3,4-5,6-7$ per week). 


\section{Statistical Analyses}

Characteristics of the sample population were described in relation to device measured sitting. Multiple Logistic Regression was used to estimate the association between SB and depression diagnosis or symptoms. Device-measured sitting was modelled using daily averages which were categorised into roughly equal tertiles ( $<8$ hours; $8-10$ hours; $>10$ hours). Self -reported SB was categorised into four roughly equal groups ( $0-1$ hours; $1-2$ hours; $2-3$ hours; $>3$ hours). Occupational activity was based on four groups (sitting occupation; standing occupation; physical work; heavy manual work). The models were adjusted for sex, device wear time, cigarette smoking, body mass index, alcohol consumption, disability, educational attainment and MVPA. All statistical analyses were performed in IBM SPSS Statistics 26.

\section{Results}

A total of 6,562 ( $88 \%$ of those invited) cohort members consented to participate in the present study. After removal of 1114 participants with unusable activPAL data (nurse unable to initiate $(n=102)$; lost in post $(n=591)$; unable to download $(n=421))$, and missing covariates $(n=742)$, the analytic sample for device measured sitting comprised 4,706 participants (52.4\% Female). Participants declining to wear the device were more likely to be male, smokers, report poorer health, and be obese. [34] The analytical sample for selfreported SB comprised 6,727 participants (52.2\% Female).

\section{Device-measured sample characteristics}

Table 1 shows that participants in the highest sitting tertile were more likely to be male, obese, report a depression diagnosis and greater depressive symptoms, to be smokers, consume alcohol on a weekly basis, have a secondary education, to have more severe disability and record the lowest amount of device measured MVPA. The prevalence of depression diagnosis and symptoms were $6.7 \%$ and $17.0 \%$ respectively, (Pearson correlation $r=0.27$ between depression diagnosis and symptoms).

Device measured sitting time, moderate-vigorous physical activity, sit to stand transitions and depression diagnosis

Compared to those who sat for $<8$ hours a day, participants who sat for longer hours had increased odds of depression diagnosis (Table 2). These associations persisted after adjustment for covariates albeit the effect estimate was attenuated. In contrast there were lower odds of depression diagnosis in participants recording $>0.9$ hours/day of MVPA, when compared to $<0.6$ hours/day (Table 2 ). However, no association was observed between device measured sit to stand transitions and depression diagnosis (Table S1). 
Device measured sitting time, moderate-vigorous physical activity, sit to stand transitions and depressive symptoms

There was some evidence of a ' $U$ '-shaped association between sitting and depressive symptoms, with participants reporting 8-10 hr/d, demonstrating lower odds of elevated symptoms (Table 2) compared to $<8$ $\mathrm{hr} / \mathrm{d}$ of sitting time. MVPA was associated with lower odds of symptoms in a dose dependent manner (Table 2). Associations of sitting and MVPA were mutually independent across all statistical models. Conversely there appeared to be no association between total number of device measured sit to stand transitions and depressive symptoms (Table S1).

Self-reported sedentary behaviours, depression diagnosis and depressive symptoms

The fully adjusted model showed no association between weekday TV viewing and depression diagnosis (Table 3). There was some evidence for elevated odds of depression with moderate (2-3hr/d) but not high (>3 hr/d) internet use (Table 4). No associations were seen for occupational activity and depression diagnosis (Table 5). Increased odds of depressive symptoms were observed in participants reporting $>3$ hours/day of TV weekday viewing time compared to $<1 \mathrm{hr} / \mathrm{d}$ (Table 3). The association persisted in covariate adjusted models, albeit it was heavily attenuated. Both moderate and high internet use was associated with elevated depressive symptoms (table 4), although no associations were seen for occupational activity and depressive symptoms (table 5).

\section{Discussion}

The aim of the current study was to investigate the associations of device-measured sitting time and selfreported sedentary behaviours with depression utilising different methodological approaches. We found an increased odds of depression diagnosis in those with higher levels of device measured sitting time, although associations with context specific sedentary behaviours and depression diagnosis were less consistent. In contrast there was no association between device measured sitting time and depressive symptoms. TV viewing and internet usage were more consistently associated with higher depressive symptoms. This raises the possibility that the self-reported associations are spurious since depressive symptoms possibly bias the way in which participants respond to questions on sedentary behaviours. Device measured MVPA was associated with lower odds of both depression diagnosis and depressive symptoms in a dose dependent manner. There was no association between the number of device measured sit to stand transitions and depression (diagnosis and symptoms).

\section{Comparison to existing literature}

Limited studies have utilised wearable devices to examine associations between sitting behaviour and depression in adults. Those that have were mostly conducted on small $(n<500)$ convenience samples. Most of these studies [35-40], but not all [15] have noted associations between device measured sitting and higher risk of depressive symptoms assessed from self-reported questionnaires. To our knowledge only one 
small population based study [40] has previously employed the gold standard thigh worn position to examine sitting time in relation to depression. Our findings on device measured MVPA with both depression symptoms and diagnosis, supports the results from some previous studies [15, 41], although others have only observed protective associations with light intensity activity and not MVPA [36-39]. It is conceivable that in older populations lighter intensity activity may be more beneficial for mental health as greater exertion during vigorous forms of exercise may produce discomfort and shortness of breath, thus feel less enjoyable.

The data on TV viewing and internet usage are consistent with the results that were synthesised from a recent meta-analysis on the association between SB and diagnosed depression, which noted an increased risk of depression diagnosis in relation to TV viewing and computer usage.[11] Our finding of a null association between the number of sit to stand transitions and depression, appears largely consistent with a recent study on three elderly Scottish cohorts. Their data showed associations between sit to stand transitions and depression in only one of the three cohorts examined. [40] Indeed the mechanisms explaining any links between active breaks and depression seem unclear.

\section{Mechanisms}

The possible explanation of an increased odds of depressive symptoms with both TV viewing and internet usage could be plausibly explained by factors such as social isolation, which is a potential cause for depression within the susceptible members of the population. $[42,43]$ However, excessive prolonged periods of SB has also been associated with biomarkers of low grade inflammation, such as C-reactive protein. Thus, biological mechanisms may also explain associations between sitting and depression. This has been further supported by a randomised controlled trial, which observed higher depressive mood in parallel with elevated inflammatory biomarkers after a week of experimentally induced SB.[44]

\section{Strengths and limitations}

The main limitation was the cross-sectional design as we cannot infer what direction the association is operating in. Bi-directional associations are not unlikely (i.e. whether sitting time is causing poor mental health or vice versa), although recent evidence using genetic instrumental variables suggested protective effects of physical activity on depression. [45] The depression outcome variables were self-reported, which could be influenced by reporting bias. Nevertheless, capturing anti-depressant usage has shown to be a valid approach for clinical depression diagnoses.[46]

A major strength of the current study is the combined use of devices and self-report tools to capture sedentary behaviour in its totality. This enabled us to capture both accurate and comprehensive information on total sitting volume and the context where such behaviours occur. The thigh worn position is considered the gold standard for free living sitting measurements. We adjusted our models for a wide range of covariates to limit possible residual confounding. Moreover, participants were all born in the same week of 1970 , therefore limiting the confounding influences of age.

\section{Conclusion}


Device measured sitting time was associated with clinically diagnosed depression. Self-reported sedentary behaviours were only associated with depressive symptoms. Encouraging physical activity and reducing sitting time could be beneficial in preventing depression.

\section{Declarations}

Ethics approval and consent to participate: Local research ethics committees approved all aspects of the survey (NRES Committee South East Coast - Brighton \& Sussex , Ref 15/LO/1446) and all participants gave written informed consent.

Consent for publication: Not applicable

Availability of data and material: The datasets generated and/or analysed during the current study are publicly available from the UK data service https://www.ukdataservice.ac.uk/

Competing interests: ES and MH received an unrestricted grant from PAL Technologies, Scotland, UK.

Funding: British Heart Foundation (SP/15/6/31397). David Batty is supported by the UK Medical Research Council (MR/P023444/1) and the US National Institute on Aging (1R56AG052519-01; 1R01AG05251901A1). Mark Hamer is funded through a joint award from Economic Social Research Council and Medical Research Council (RES-579-47-0001). The funders had no role in the study design; in the collection, analysis and interpretation of data; in writing of the report; or in the decision to submit the paper for publication.

Authors' contributions: MH/ES obtained funding, conceptualized and designed the study. AW performed analyses, drafted the initial manuscript. MH and GDB supervised the analyses and write-up. NP analysed Activpal data. All authors contributed to critical revision of paper and approved the final manuscript as submitted. MH is the manuscript's guarantor.

Acknowledgements: N/A

\section{References}

1. Baker C. Mental health problems: statistics on prevalence and services. 2018; Available from: http://researchbriefings.parliament.uk/ResearchBriefing/Summary/SN06988

2. WHO. Depression: A Global Crisis. World Ment Heal Day [Internet]. 2012; from: http://www.who.int/mental_health/management/depression/wfmh_paper_depression_wmhd_2012.pdf

3. Batty GD, Kivimäki M, Bell S, Gale CR, Shipley M, Whitley E, et al. Psychosocial characteristics as potential predictors of suicide in adults: An overview of the evidence with new results from prospective cohort studies. Transl Psychiatry 2018;8.

4. McAninch J, Greene C, Sorkin JD, Lavoie MC, Smith GS. Higher psychological distress is associated with unintentional injuries in US adults. Inj Prev. 2014;20:258-65.

5. Hert M De, Detraux J, Vancampfort D. The intriguing relationship between coronary heart disease and mental disorders. Dialogues Clin Neurosci. 2018;20:31-40. 
6. Batty GD, Russ TC, Stamatakis E, Kivimäki M. Psychological distress in relation to site specific cancer mortality: Pooling of unpublished data from 16 prospective cohort studies. BMJ. 2017;356.

7. Mammen G, Faulkner G. Physical activity and the prevention of depression: A systematic review of prospective studies. Am J Prev Med 2013;45:649-57.

8. Schuch FB, Vancampfort D, Firth J, Rosenbaum S, Ward PB, Silva ES, et al. Physical activity and incident depression: A meta-analysis of prospective cohort studies. Am J Psychiatry. 2018;175:631-48.

9. Tremblay MS, Aubert S, Barnes JD, Saunders TJ, Carson V, Latimer-Cheung AE, et al. Sedentary Behavior Research Network (SBRN) - Terminology Consensus Project process and outcome. Int J Behav Nutr Phys Act. 2017;14:1-17.

10. Huang Y, Li L, Gan Y, Wang C, Jiang H, Cao S, et al. Sedentary behaviors and risk of depression: a metaanalysis of prospective studies. Transl Psychiatry 2020;10(1):26.

11. Zhai L, Zhang Y, Zhang D. Sedentary behaviour and the risk of depression: A meta-analysis. Br J Sports Med. 2015;49:705-9.

12. Edwards MK, Loprinzi PD. Effects of a Sedentary Behavior-Inducing Randomized Controlled Intervention on Depression and Mood Profile in Active Young Adults. Mayo Clin Proc 2016;91:984-98.

13. Atkin AJ, Gorely T, Clemes SA, Yates T, Edwardson C, Brage S, et al. Methods of measurement in epidemiology: Sedentary behaviour. Int J Epidemiol. 2012; 41(5):1460-71

14. Voncken MJ, Bögels SM, Peeters F. Specificity of interpretation and judgemental biases in social phobia versus depression. Psychol Psychother Theory Res Pract. 2007;80:443-53.

15. Vallance JK, Winkler EAH, Gardiner PA, Healy GN, Lynch BM, Owen N. Associations of objectivelyassessed physical activity and sedentary time with depression: NHANES (2005-2006). Prev Med 2011;53:284-8.

16. Thomée S, Härenstam A, Hagberg M. Computer use and stress, sleep disturbances, and symptoms of depression among young adults - a prospective cohort study. BMC Psychiatry. 2012;12.

17. Peeters GMEEG, Burton NW, Brown WJ. Associations between sitting time and a range of symptoms in mid-age women. Prev Med 2013;56:135-41.

18. Chastin SFM, Dontje ML, Skelton DA, Čukić I, Shaw RJ, Gill JMR, et al. Systematic comparative validation of self-report measures of sedentary time against an objective measure of postural sitting (activPAL). Int J Behav Nutr Phys Act. 2018;15(1):21.

19. Elliott J, Shepherd P. Cohort profile: 1970 British Birth Cohort (BCS70). Int J Epidemiol. 2006;35:836-43.

20. University of London loE, Centre for Longitudinal Studies. 1970 British Cohort Study: Forty-Six-Year Follow-Up, 2016-2018 (http://doi.org/10.5255/UKDA-SN-8547-1): UK Data Service; 2019.

21. Stansfield B, Hajarnis M, Sudarshan R. Characteristics of very slow stepping in healthy adults and validity of the activPAL3 ${ }^{\mathrm{TM}}$ activity monitor in detecting these steps. Med Eng Phys 2015;37:42-7.

22. Kozey-Keadle S, Libertine A, Lyden K, Staudenmayer J, Freedson PS. Validation of wearable monitors for assessing sedentary behavior. Med Sci Sports Exerc. 2011;43:1561-7.

23. Lyden K, Kozey Keadle SL, Staudenmayer JW, Freedson PS. Validity of two wearable monitors to estimate breaks from sedentary time. Med Sci Sports Exerc. 2012;44:2243-52. 
24. Grant PM, Ryan CG, Tigbe WW, Granat MH. The validation of a novel activity monitor in the measurement of posture and motion during everyday activities. Br J Sports Med. 2006;40:992-7.

25. Ryan CG, Grant PM, Tigbe WW, Granat MH. The validity and reliability of a novel activity monitor as a measure of walking. Br J Sports Med. 2006;40:779-84.

26. Kim Y, Barry VW, Kang M. Validation of the ActiGraph GT3X and activPAL Accelerometers for the Assessment of Sedentary Behavior. Meas Phys Educ Exerc Sci 2015;19:125-37.

27. Dall PM, Skelton DA, Dontje ML, Coulter EH, Stewart S, Cox SR, et al. Characteristics of a Protocol to Collect Objective Physical Activity/Sedentary Behavior Data in a Large Study: Seniors USP (Understanding Sedentary Patterns). J Meas Phys Behav. 2018;1:26-31.

28. Winkler EAH, Bodicoat DH, Healy GN, Bakrania K, Yates T, Owen N, et al. Identifying adults' valid waking wear time by automated estimation in activPAL data collected with a $24 \mathrm{~h}$ wear protocol. Physiol Meas. 2016;37:1653-68.

29. Tudor-Locke C, Aguiar EJ, Han H, Ducharme SW, Schuna JM, Barreira T V., et al. Walking cadence (steps/min) and intensity in 21-40 year olds: CADENCE-adults. Int J Behav Nutr Phys Act. 2019;16:111.

30. Rutter M, Tizard J, Whitmore K. Education, health and behaviour. London: Longmans; 1970.

31. McGee R, Williams S, Silva PA. An evaluation of the Malaise inventory. J Psychosom Res. 1986;30:147-52.

32. Rodgers B, Pickles A, Power C, Collishaw S, Maughan B. Validity of the Malaise Inventory in general population samples. Soc Psychiatry Psychiatr Epidemiol. 1999;34:333-41.

33. Ploubidis GB, McElroy E, Moreira HC. A longitudinal examination of the measurement equivalence of mental health assessments in two british birth cohorts. Longit Life Course Stud. 2019;10:471-89.

34. Hamer M, Stamatakis E, Chastin S, Pearson N, Brown M, Gilbert E, et al. Feasibility of Measuring Sedentary Time with Thigh Worn Accelerometry and Sociodemographic Correlates: The 1970 British Cohort Study. Am J Epidemiol [in press]

35. Brazendale K, Drenowatz C, Falck RS, Randel AB, Hoffmeyer JD, Hand GA, et al. Depressive Symptoms Are Positively Associated with Time Spent Sedentary in Healthy Young US Adults. Prog Prev Med. 2017;2:e0004.

36. Yasunaga A, Shibata A, Ishii K, Koohsari MJ, Oka K. Cross-sectional associations of sedentary behaviour and physical activity on depression in Japanese older adults: An isotemporal substitution approach. BMJ Open. 2018;8:6-10.

37. Chu AHY, van Dam RM, Biddle SJH, Tan CS, Koh D, Müller-Riemenschneider F. Self-reported domainspecific and accelerometer-based physical activity and sedentary behaviour in relation to psychological distress among an urban Asian population. Int J Behav Nutr Phys Act. 2018;15:1-14.

38. Dillon CB, McMahon E, O’Regan G, Perry IJ. Associations between physical behaviour patterns and levels of depressive symptoms, anxiety and well-being in middle-aged adults: A cross-sectional study using isotemporal substitution models. BMJ Open. 2018;8:1-8. 
39. Hamer M, Coombs N, Stamatakis E. Associations between objectively assessed and self-reported sedentary time with mental health in adults: an analysis of data from the Health Survey for England. BMJ Open. 2014;4(3):e004580.

40. Okely JA, Čukić I, Shaw RJ, Chastin SF, Dall PM, Deary IJ, et al. Positive and negative well-being and objectively measured sedentary behaviour in older adults: Evidence from three cohorts. BMC Geriatr. 2019;19:1-10.

41. Loprinzi PD. Objectively measured light and moderate-to-vigorous physical activity is associated with lower depression levels among older US adults. Aging Ment Heal 2013;17:801-5.

42. Kraut R, Patterson M, Lundmark V, Kiesler S, Mukophadhyay T, Scherlis W. Internet paradox: A social technology that reduces social involvement and psychological well-being? Am Psychol. 1998;53:101731.

43. Beutel ME, Klein EM, Brähler E, Reiner I, Jünger $C$, Michal M, et al. Loneliness in the general population: Prevalence, determinants and relations to mental health. BMC Psychiatry 2017;17:1-7.

44. Endrighi R, Steptoe A, Hamer M. The effect of experimentally induced sedentariness on mood and psychobiological responses to mental stress. Br J Psychiatry. 2016;208:245-51.

45. Choi KW, Chen CY, Stein MB, Klimentidis YC, Wang MJ, Koenen KC, Smoller JW; Major Depressive Disorder Working Group of the Psychiatric Genomics Consortium. Assessment of Bidirectional Relationships Between Physical Activity and Depression Among Adults: A 2-Sample Mendelian Randomization Study. JAMA Psychiatry. 2019 Apr 1;76(4):399-408.

46. Lucas M, Mekary R, Pan A, Mirzaei F, O'Reilly ÉJ, Willett WC, et al. Relation between clinical depression risk and physical activity and time spent watching television in older women: A 10-year prospective follow-up study. Am J Epidemiol. 2011;174:1017-27.

\section{Tables}

Table 1: Characteristics of the study sample according to average sitting time at age 46, The British Birth Cohort Study 


\begin{tabular}{|c|c|c|c|}
\hline \multirow[b]{2}{*}{ Characteristic } & \multicolumn{3}{|c|}{ Average sitting time (Hours/day) } \\
\hline & $\begin{array}{c}<8 \\
(n=1,524)\end{array}$ & $\begin{array}{c}8-10 \\
(n=1,561)\end{array}$ & $\begin{array}{c}>10 \\
(n=1,521)\end{array}$ \\
\hline \multicolumn{4}{|l|}{ Sex $(\%)$} \\
\hline Male & 39.9 & 46.3 & 55.8 \\
\hline Female & 60.1 & 53.7 & 44.2 \\
\hline \multicolumn{4}{|c|}{ Doctor Depression and Anti-depressant Usage (\%) } \\
\hline Yes & 5.4 & 6.0 & 8.7 \\
\hline No & 94.6 & 94.0 & 91.3 \\
\hline \multicolumn{4}{|l|}{ Malaise Score (\%) } \\
\hline High $\geq 4$ & 17.3 & 14.6 & 19.3 \\
\hline Low $<4$ & 82.7 & 85.4 & 80.7 \\
\hline \multicolumn{4}{|l|}{ Cigarette Smoking (\%) } \\
\hline Never & 50.9 & 51.5 & 48.6 \\
\hline Ex-Smoker & 31.2 & 32.5 & 32.7 \\
\hline Occasional Smoker & 5.2 & 4.9 & 4.0 \\
\hline Daily Smoker & 12.7 & 11.0 & 14.7 \\
\hline \multicolumn{4}{|l|}{ Alcohol Frequency (\%) } \\
\hline Never & 10.6 & 9.4 & 9.3 \\
\hline Monthly & 41.9 & 41.7 & 44.0 \\
\hline Weekly & 47.6 & 48.9 & 46.7 \\
\hline \multicolumn{4}{|l|}{ Body Mass Index (\%) } \\
\hline Underweight & 1.0 & 0.3 & 0.7 \\
\hline Normal Weight & 35.0 & 28.8 & 23.5 \\
\hline Overweight & 35.9 & 39.4 & 39.1 \\
\hline Obese & 28.0 & 31.6 & 36.8 \\
\hline \multicolumn{4}{|l|}{ Education (\%) } \\
\hline No Qualification & 28.7 & 23.3 & 25.2 \\
\hline Secondary Education & 49.3 & 45.7 & 44.5 \\
\hline Graduate Education & 21.9 & 31.0 & 30.3 \\
\hline \multicolumn{4}{|l|}{ Disability (\%) } \\
\hline No & 87.0 & 85.9 & 82.1 \\
\hline Disabled to Some Extent & 10.4 & 9.0 & 10.6 \\
\hline Severely Disabled & 2.6 & 5.0 & 7.4 \\
\hline \multicolumn{4}{|l|}{ MVPA hr/d (\%) } \\
\hline$<0.6$ & 19.6 & 30.4 & 48.1 \\
\hline $0.6-0.9$ & 33.1 & 36.0 & 32.3 \\
\hline$>0.9$ & 47.3 & 33.6 & 19.5 \\
\hline
\end{tabular}

Malaise Score defined as

a $\geq 4$ score defined a high malaise inventory score

${ }^{\mathrm{b}}$ 0-3 Score defined a low malaise inventory score

Body mass index classified as

${ }^{c} 0-18.49 \mathrm{~kg} / \mathrm{m}^{2}$

d $18.5-24.9 \mathrm{~kg} / \mathrm{m}^{2}$

e $25.0-29.9 \mathrm{~kg} / \mathrm{m}^{2}$

f $\geq 30.0 \mathrm{~kg} / \mathrm{m}^{2}$

Education defined as

g No formal educational attainment

h Participant has either GCSEs, A-Levels or a Diploma

I Graduate Education; Bachelors, Masters and Doctoral Degrees

Disability defined as

j No EU-SILC longstanding health condition

${ }^{\mathrm{k}}$ EU SILC classification to some extent 
${ }^{\mathrm{L}}$ EU SILC classification severely hampered

Table 2: Device measured sitting time and moderate-vigorous physical activity in relation to depression diagnosis and symptoms, the British Birth Cohort Study

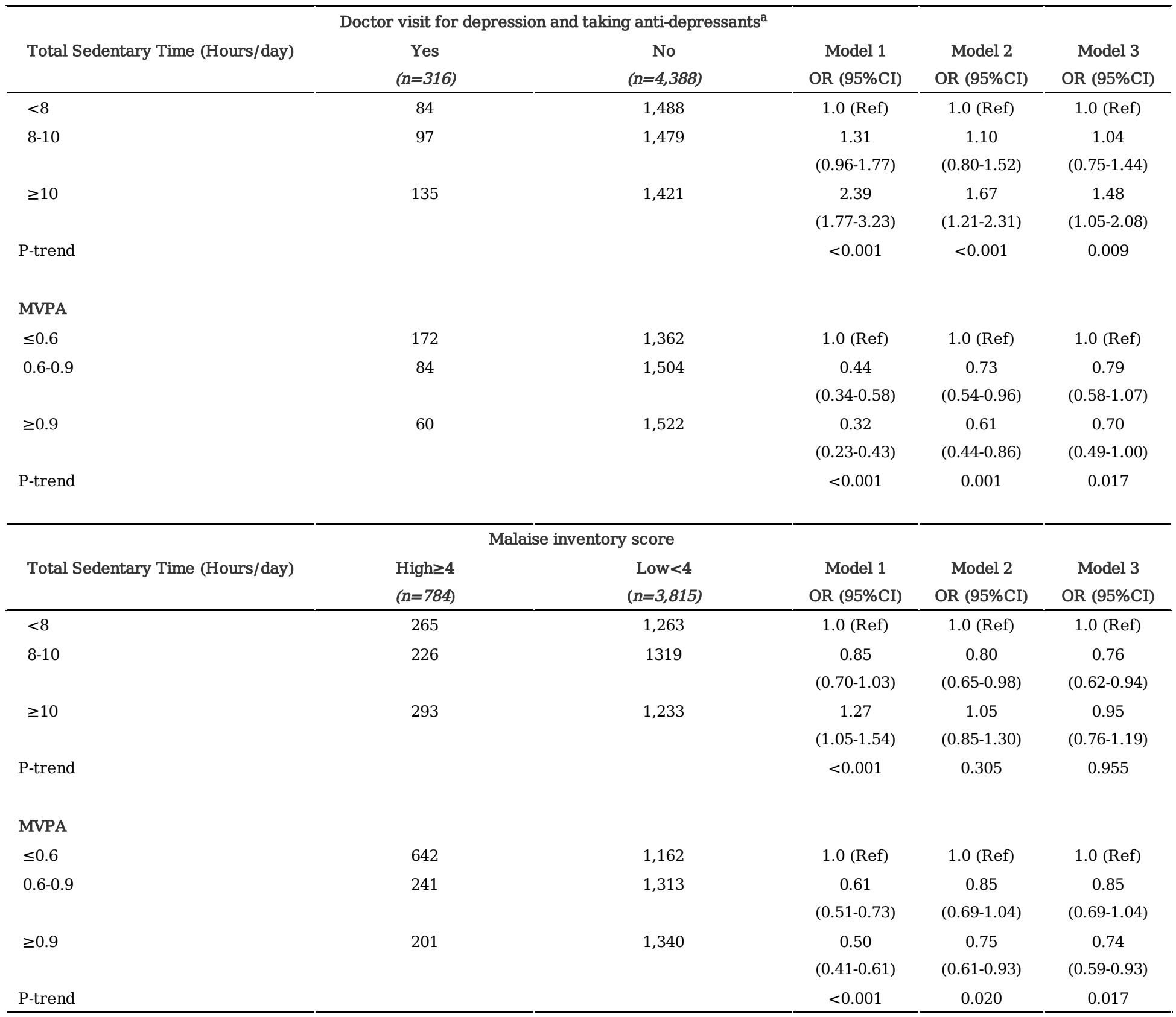

Model adjusted for

Model 1 - sex, device waking wear time (Hours)

Model 2 - sex, device waking wear time (Hours), cigarette smoking, body mass index, alcohol consumption, disability, education

Model 3 - sex, waking wear time (Hours), cigarette smoking, body mass index, alcohol consumption, disability, education, mutually for MVPA or sedentary

a Depression diagnoses was established via the combination of reported doctor visit for depression and reported taking a prescription of antidepressants 
Table 3: TV weekday viewing time in relation to depression diagnosis and malaise inventory score, the British Birth Cohort Study

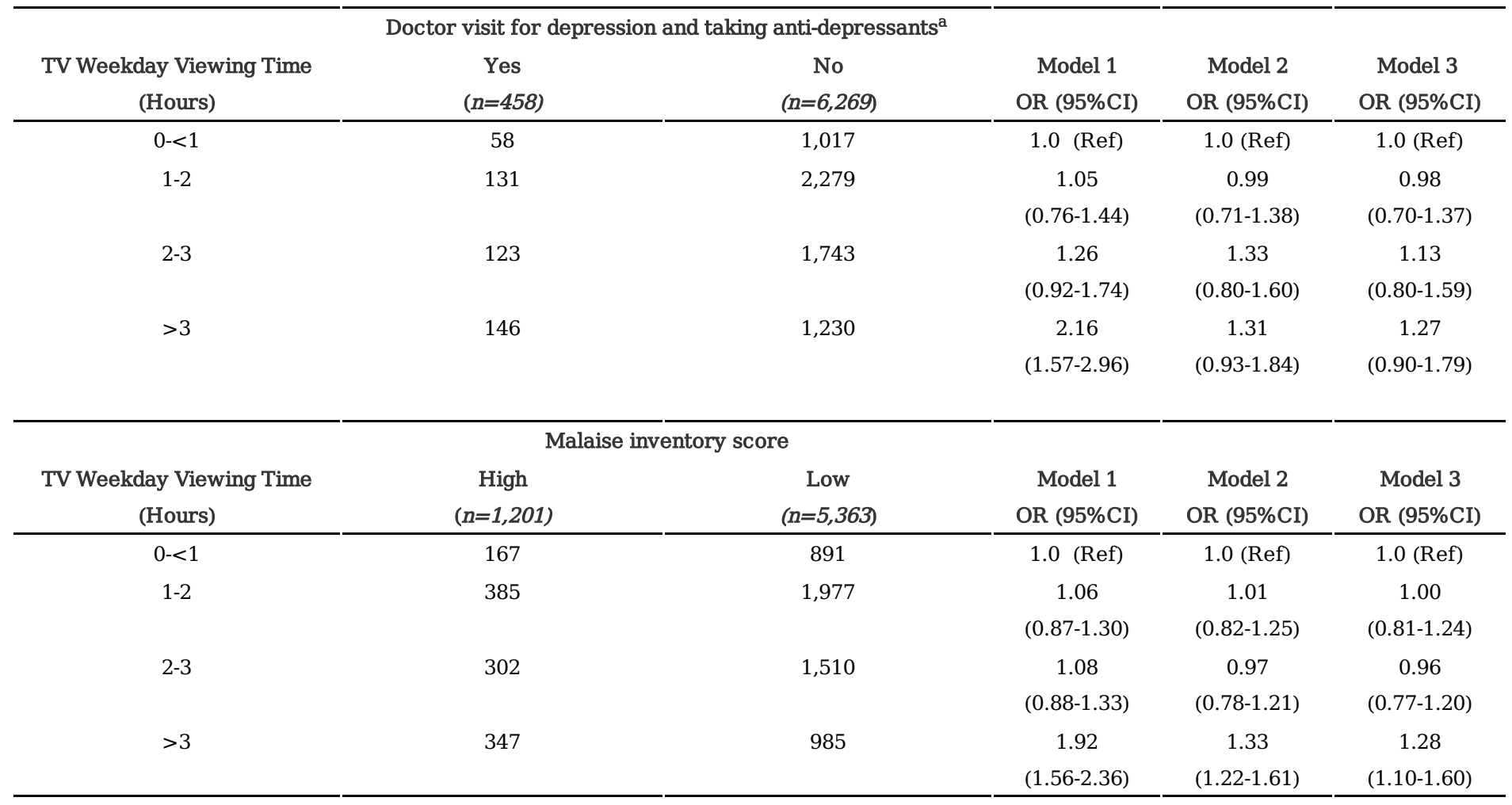

Model adjusted for

Model 1 - sex

Model 2 - sex, cigarette smoking, body mass index, alcohol consumption, disability, education

Model 3 - sex, cigarette smoking, body mass index, alcohol consumption, disability, education, self-reported exercise

a Depression diagnoses was established via the combination of reported doctor visit for depression and reported taking a prescription of antidepressants

Table 4: Internet average weekday usage in relation to depression diagnosis and malaise inventory score, the British Birth Cohort Study

Table 5: Occupational work activity type in relation to depression diagnosis and malaise inventory score, the British Birth Cohort 


\begin{tabular}{|c|c|c|c|c|c|}
\hline \multirow[b]{2}{*}{$\begin{array}{l}\text { Internet average weekday } \\
\text { usage (Hours) }\end{array}$} & \multicolumn{2}{|c|}{$\begin{array}{l}\text { Doctor visit for depression and taking anti- } \\
\qquad \text { depressants }^{\mathrm{a}}\end{array}$} & \multirow[b]{2}{*}{$\begin{array}{c}\text { Model } 1 \\
\text { OR } \\
(95 \% \mathrm{CI}) \\
\end{array}$} & \multirow[b]{2}{*}{$\begin{array}{c}\text { Model } 2 \\
\text { OR (95\%CI) }\end{array}$} & \multirow[b]{2}{*}{$\begin{array}{c}\text { Model } 3 \\
\text { OR (95\%CI) }\end{array}$} \\
\hline & $\begin{array}{c}\text { Yes } \\
(n=456)\end{array}$ & $\begin{array}{c}\text { No } \\
(n=6,267)\end{array}$ & & & \\
\hline $0-<1$ & 142 & 2,343 & 1.0 (Ref) & 1.0 (Ref) & 1.0 (Ref) \\
\hline $1-2$ & 168 & 2,468 & $\begin{array}{c}1.14 \\
(0.91-1.44)\end{array}$ & $\begin{array}{c}1.19 \\
(0.94-1.52)\end{array}$ & $\begin{array}{c}1.19 \\
(0.93-1.52)\end{array}$ \\
\hline $2-3$ & 76 & 778 & $\begin{array}{c}1.62 \\
(1.21-2.17)\end{array}$ & $\begin{array}{c}1.52 \\
(1.12-2.07)\end{array}$ & $\begin{array}{c}1.52 \\
(1.12-2.07)\end{array}$ \\
\hline \multirow[t]{2}{*}{$>3$} & 70 & 678 & $\begin{array}{c}1.72 \\
(1.31-2.39)\end{array}$ & $\begin{array}{c}1.17 \\
(0.84-1.62)\end{array}$ & $\begin{array}{c}1.14 \\
(0.82-1.59)\end{array}$ \\
\hline & \multicolumn{2}{|c|}{ Malaise inventory score } & & & \\
\hline $\begin{array}{l}\text { Internet average weekday } \\
\text { usage (Hours) }\end{array}$ & $\begin{array}{c}\text { High } \\
(n=1,119)\end{array}$ & $\begin{array}{c}\text { Low } \\
(n=5,363)\end{array}$ & $\begin{array}{c}\text { Model } 1 \\
\text { OR } \\
(95 \% \mathrm{CI}) \\
\end{array}$ & $\begin{array}{c}\text { Model } 2 \\
\text { OR (95\%CI) }\end{array}$ & $\begin{array}{c}\text { Model } 3 \\
\text { OR (95\%CI) }\end{array}$ \\
\hline $0-<1$ & 380 & 2,039 & 1.0 (Ref) & 1.0 (Ref) & 1.0 (Ref) \\
\hline $1-2$ & 427 & 2,152 & $\begin{array}{c}1.08 \\
(0.92-1.25)\end{array}$ & $\begin{array}{c}1.11 \\
(0.95-1.30)\end{array}$ & $\begin{array}{c}1.11 \\
(0.95-1.30)\end{array}$ \\
\hline $2-3$ & 195 & 639 & $\begin{array}{c}1.65 \\
(1.35-2.00)\end{array}$ & $\begin{array}{c}1.60 \\
(1.30-1.97)\end{array}$ & $\begin{array}{c}1.60 \\
(1.30-1.97)\end{array}$ \\
\hline$>3$ & 197 & 533 & $\begin{array}{c}2.04 \\
(1.67-2.49)\end{array}$ & $\begin{array}{c}1.67 \\
(1.35-2.07)\end{array}$ & $\begin{array}{c}1.63 \\
(1.32-2.03)\end{array}$ \\
\hline
\end{tabular}

Model adjusted for

Model 1 - sex

Model 2 - sex, cigarette smoking, body mass index, alcohol consumption, disability, education

Model 3 - sex, cigarette smoking, body mass index, alcohol consumption, disability, education, self-reported exercise

a Depression diagnoses was established via the combination of reported doctor visit for depression and reported taking a prescription of antidepressants

\section{Supplementary Files}

This is a list of supplementary files associated with this preprint. Click to download.

- OnlineSupplementaryTables.docx

- IJBNPASTROBEChecklistWebsteretal.doc 


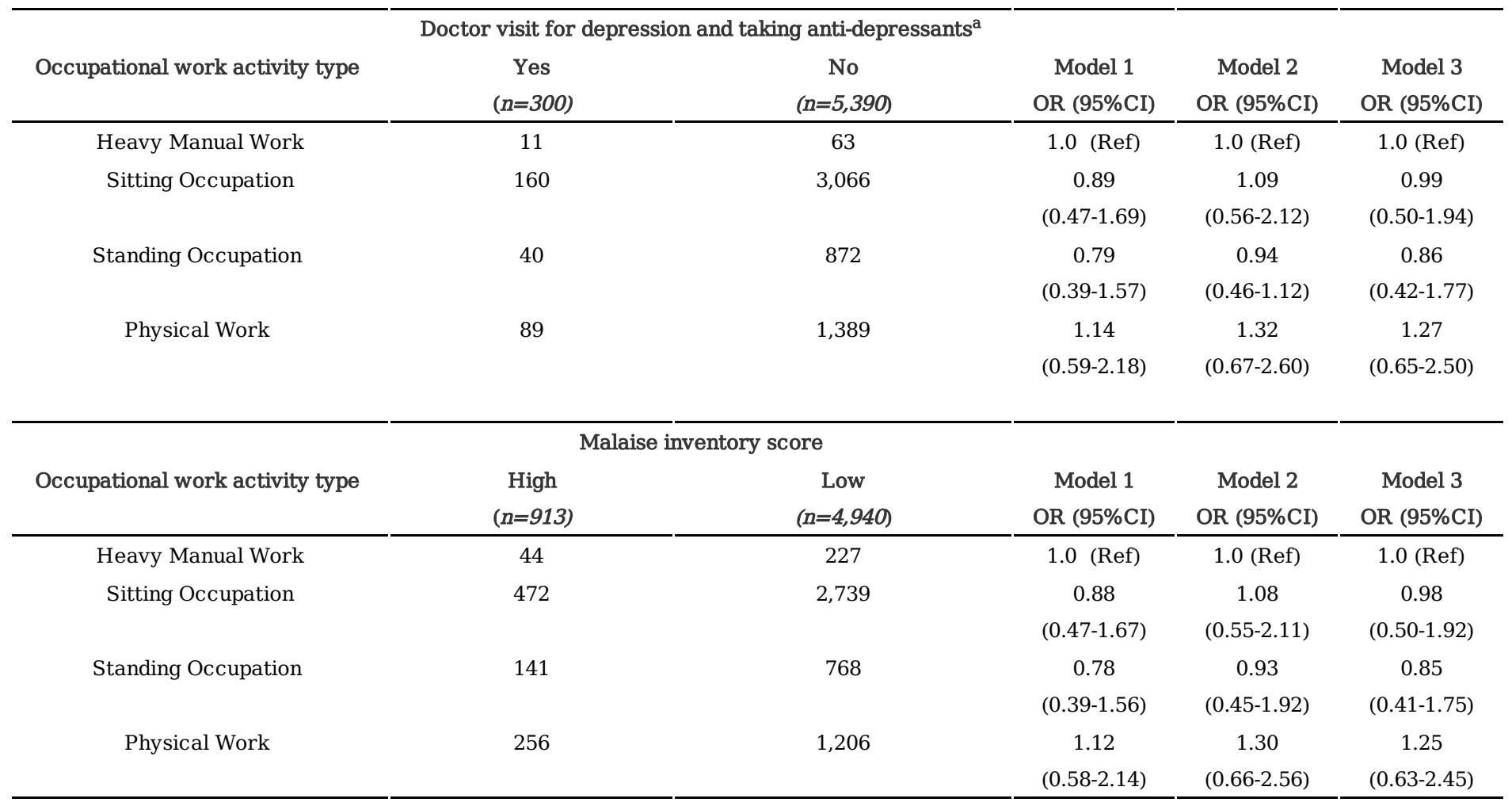

Model adjusted for

Model 1 - sex

Model 2 - sex, cigarette smoking, body mass index, alcohol consumption, disability, education

Model 3 - sex, cigarette smoking, body mass index, alcohol consumption, disability, education, self-reported exercise

${ }^{a}$ Depression diagnoses was established via the combination of reported doctor visit for depression and reported taking a prescription of antidepressants 